Supporting Information for

\title{
Urban Air Pollution Mapping Using Fleet Vehicles as Mobile Monitors and Machine Learning
}

Bu Zhao ${ }^{1,2}$, Long $\mathrm{Yu}^{3}$, Chunyan Wang ${ }^{4}$, Chenyang Shuai ${ }^{1,2}$, Ji Zhu ${ }^{5}$, Shen $\mathrm{Qu}^{6,7}$, Morteza Taiebat ${ }^{1,8}$, Ming Xu ${ }^{1,8, *}$

1. School for Environment and Sustainability, University of Michigan, Ann Arbor, MI, USA

2. Michigan Institute for Computational Discovery \& Engineering, University of Michigan, Ann Arbor, MI, USA

3. Department of Statistics, Fudan University, Shanghai, China

4. School of Environment, Tsinghua University, Beijing, China

5. Department of Statistics, University of Michigan, Ann Arbor, MI, USA

6. School of Management and Economics, Beijing Institute of Technology, Beijing, China

7. Center for Energy \& Environmental Policy Research, Beijing Institute of Technology, Beijing, China

8. Department of Civil and Environmental Engineering, University of Michigan, Ann Arbor, MI, USA

*Corresponding author: mingxu@umich.edu

\section{This PDF file includes:}

Supporting Results

Table S1-2

Fig. S1-13 


\section{Supplementary Results:}

Hyperparameters of data transformation models. Model hyperparameters were optimized by choosing the set that minimizes RMSE in 10-fold cross-validation. For the XGBoost model, eta(step size of each boosting step) $=0.1$, max_depth(maximum depth of the tree) $=5$, and nround(the max number of iterations) $=300$ are used for the transformation model for the firststage data; eta (step size of each boosting step) $=0.08$, max_depth (maximum depth of the tree) $=8$, and nround (the max number of iterations) $=500$ were used for the transformation model for the second-stage data. For the RF model, mtry (number of variables randomly sampled as candidates at each split) $=14$, ntree (number of trees to grow) $=500$ were used. For the lasso regression, lamda $=0.0647218$ were used. For ridge regression, lamda $=1.719068$ were used. For the SVR model, gamma (parameter needed for the kernel) $=0.05$, cost (cost of constraints violation) $=5$ were used. For the ANN model, there are one input layer (64 neurons) and two hidden layers (16 neurons each); the activation function is selected as "relu" and the optimizer is "RMSprop". The XGBoost, RF, SVR, and ANN models were trained using the "randomForest", "xgboost", "glmnet", "e1071", and "keras" packages in R, respectively.

Hyperparameters of $\mathbf{P M}_{2.5}$ concentration inference model. For the XGBoost model, eta (step size of each boosting step) $=0.1$, max_depth (maximum depth of the tree) $=5$, nround (the $\max$ number of iterations) $=300$ were used. For the RF model, mtry (number of variables randomly sampled as candidates at each split) $=8$, ntree (number of trees to grow) $=500$ were used. The $\mathrm{SI}$ model was based on inverse distance weighting. For the KNN model, the number of nearest neighbors (mobile monitors) were seleted as 4. For the SVR model, gamma (parameter needed for the kernel) $=0.05$, cost (cost of constraints violation) $=5$ were used. For the ANN model, there are one input layer (64 neurons) and two hidden layers (16 neurons each). The activation function is selected as "relu" and the optimizer is "RMSprop". The XGBoost, RF, SVR, and ANN models were trained using the "randomForest", "xgboost", "e1071" and "keras" packages in R, respectively.

Model evaluation metrics. The following metrics are considered to evaluate the performances of models, where $y_{i}$ is the true value, $\hat{y}_{i}$ is the prediction value and $\bar{y}$ is the sample mean:

\section{Person Correlation Coefficient :}

$$
\rho=\frac{\operatorname{COV}(\hat{Y}, Y)}{\sqrt{\operatorname{Var}[\hat{Y}] \operatorname{Var}[Y]}}
$$

Coefficient of Determination $\left(R^{2}\right)$ :

$$
R^{2}=1-\frac{\sum_{i=1}^{n}\left(y_{i}-\hat{y}_{i}\right)^{2}}{\sum_{i=1}^{n}\left(y_{i}-\bar{y}\right)^{2}}
$$

Mean Absolute Error (MAE):

$$
M A E=\frac{\sum_{i=1}^{n}\left|y_{i}-\hat{y}_{i}\right|}{n}
$$

Root Mean Square Error (RMSE):

$$
R M S E=\sqrt{\frac{\sum_{i=1}^{n}\left(y_{i}-\hat{y}_{i}\right)^{2}}{n}}
$$




\section{Supplementary tables:}

Table S1. Summary of data used in this study.

\begin{tabular}{|c|c|c|}
\hline Data Category & Data Type & Note \\
\hline $\begin{array}{l}\text { Stationary mornitoring } \\
\text { data }\end{array}$ & $\mathrm{PM}_{2.5}$ hourly concentration $\left(\mu \mathrm{g} / \mathrm{m}^{3}\right)$ & 22 stations in the study area \\
\hline \multirow[t]{5}{*}{ Meteorology } & Pressure $(\mathrm{kPa})$ & \multirow{5}{*}{$\begin{array}{l}\text { Hourly meteorological information from } \\
35 \text { stations }\end{array}$} \\
\hline & Temperature $\left({ }^{\circ} \mathrm{C}\right)$ & \\
\hline & Relative humidity (\%) & \\
\hline & Wind speed $(\mathrm{m} / \mathrm{s})$ & \\
\hline & Wind direction & \\
\hline \multirow[t]{5}{*}{ Road network } & Primary road $(\mathrm{m})$ & \multirow{5}{*}{$\begin{array}{l}\text { GIS data collected from OpenStreetMap } \\
\text { (OSM) }\end{array}$} \\
\hline & Secondary road (m) & \\
\hline & Tertiary road $(\mathrm{m})$ & \\
\hline & Motorway road (m) & \\
\hline & Trunk road $(\mathrm{m})$ & \\
\hline Land use & $\begin{array}{l}\text { Areas of land in each of the } 35 \\
\text { types }\left(\mathrm{m}^{2}\right)\end{array}$ & \\
\hline
\end{tabular}

Table S2. Model performance for data transformation.

\begin{tabular}{|llll|}
\hline Methods & MAE & RMSE & $\mathrm{R}^{2}$ \\
\hline Extreme gradient boosting (XGBoost) & 7.2 & 8.8 & 0.76 \\
Random forest (RF) & 8.5 & 12.7 & 0.60 \\
Lasso regression & 11.3 & 16.2 & 0.15 \\
Ridge regression & 11.4 & 16.3 & 0.12 \\
Support vector regression (SVR) & 8.6 & 13.1 & 0.71 \\
Artificial neural network (ANN) & 10.7 & 15.9 & 0.57 \\
\hline
\end{tabular}




\section{Supplementary figures:}

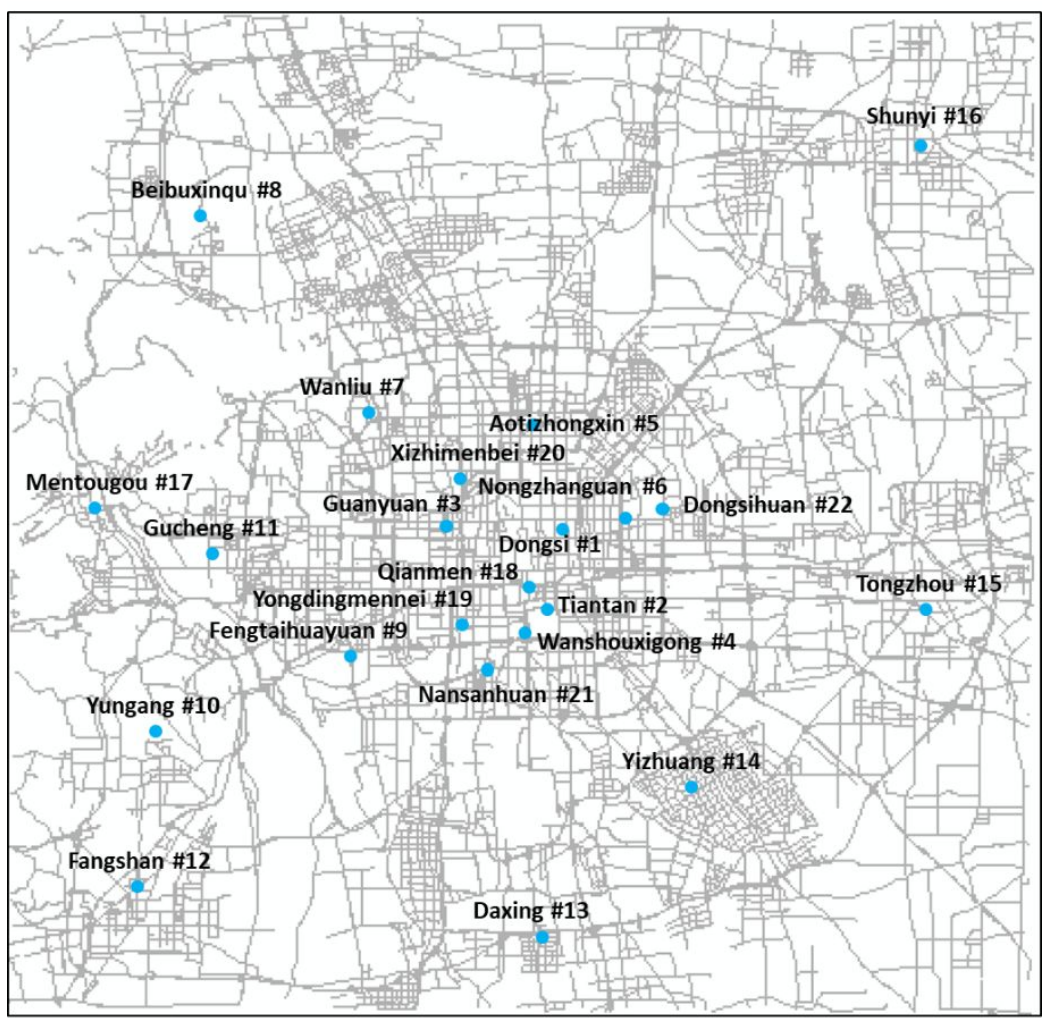

Fig. S1. Study area and the location of fixed-site monitoring stations. The study area covers all areas within the Sixth Ring Road in Beijing with about $3,363 \mathrm{~km}^{2}$. A total of 22 regulatory air quality monitoring stations are located in this area.

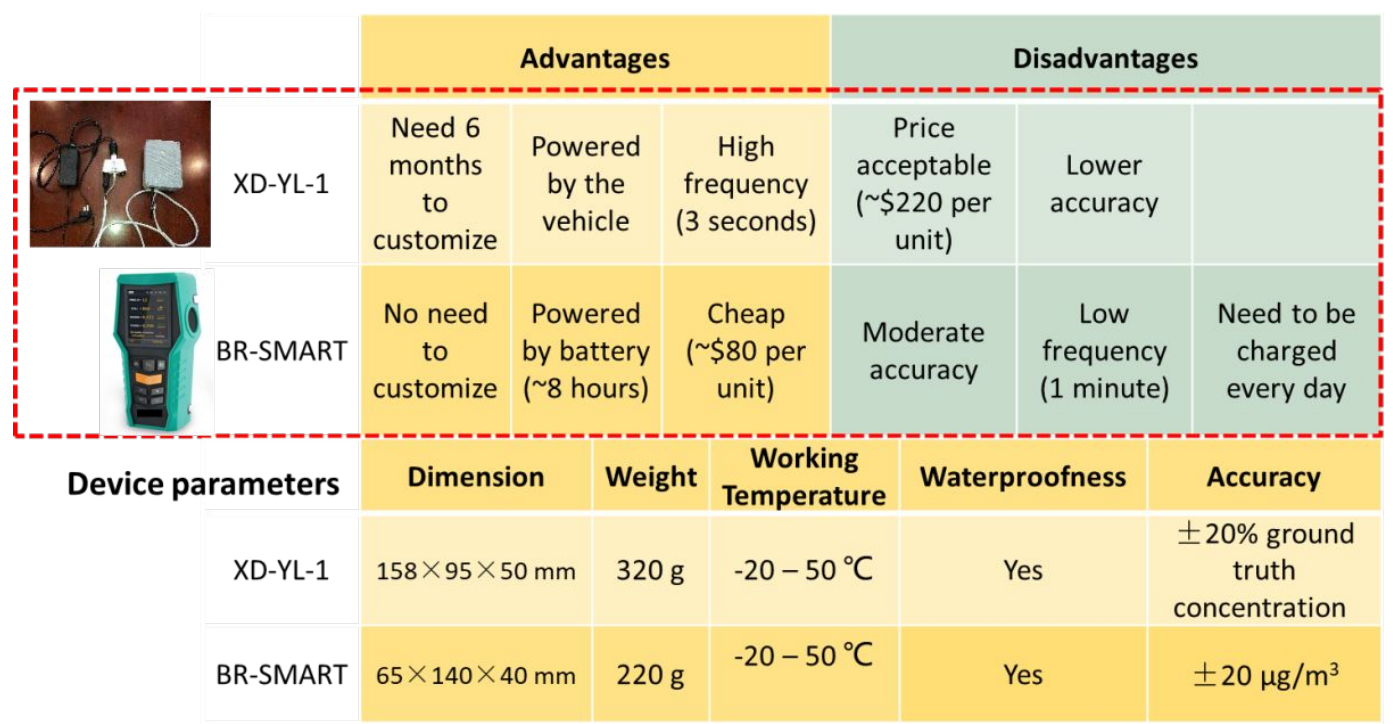

Fig. S2. Device parameters and their advantages and disadvantages. 


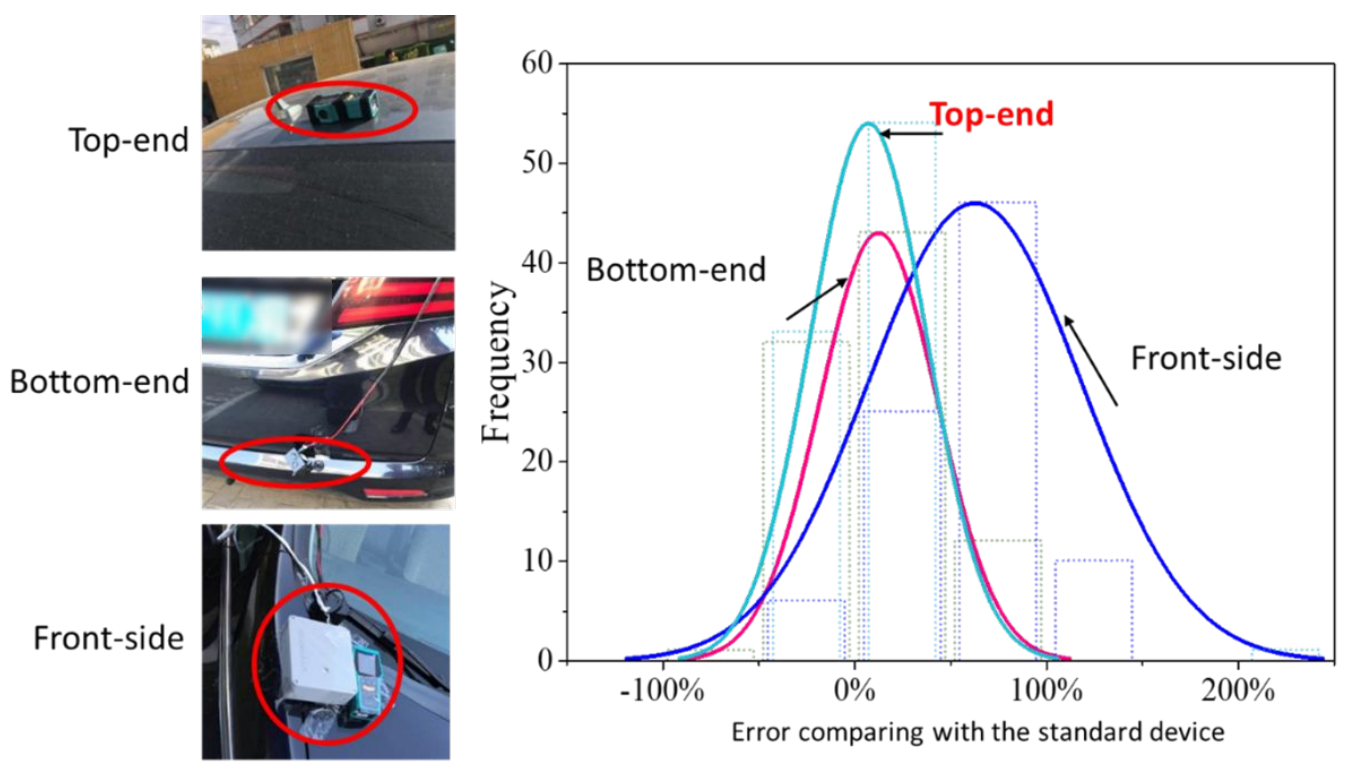

Fig. S3. Device installation methods and effects on data accuracy.

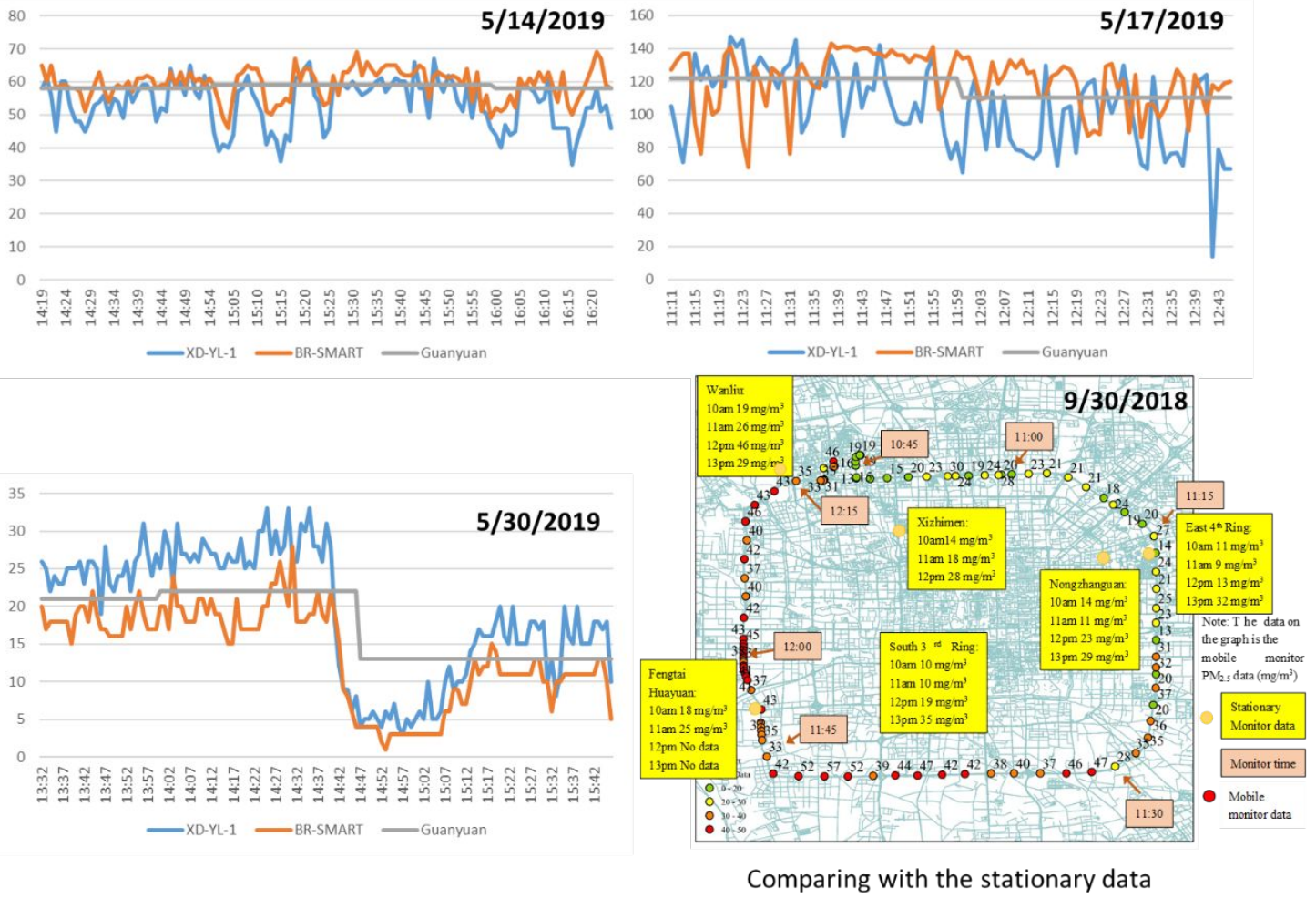

Fig. S4. Comparison of $\mathrm{PM}_{2.5}$ concentration data from the two mobile sensors and collocated stationary monitors. 


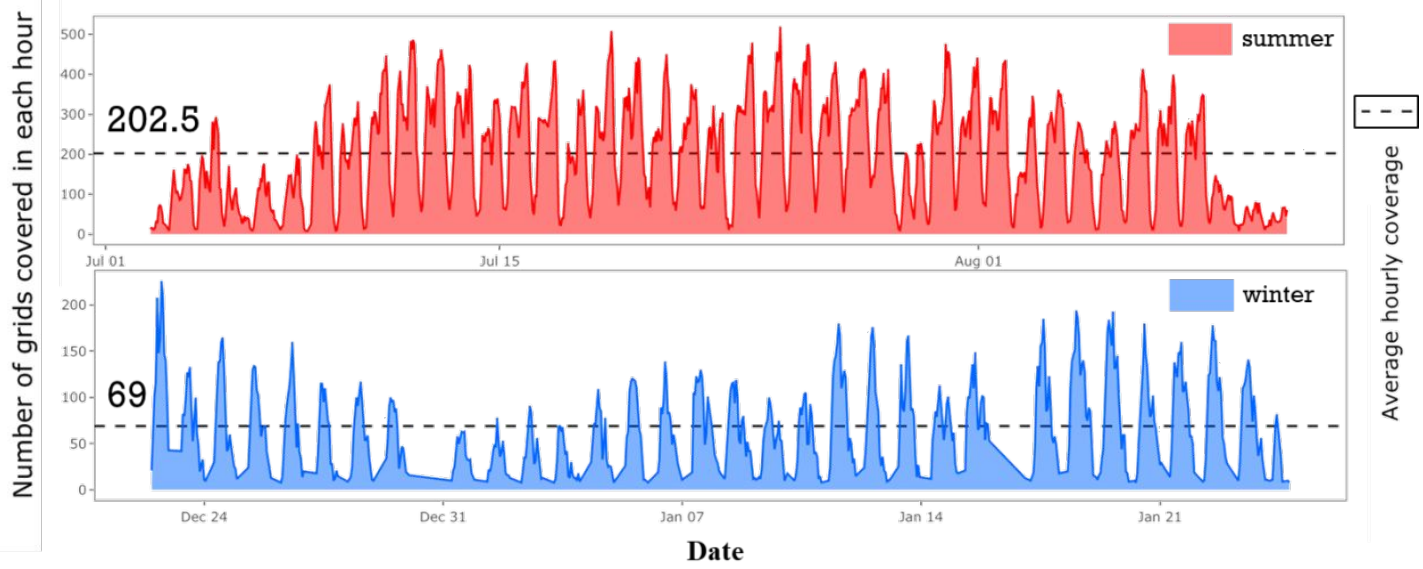

Fig. S5. Temporal characteristics for the mobile monitoring data. The mobile monitoring data cover all the time slots during the study periods. The hourly mobile monitoring results generally show a bimodal distribution (peak at 14:00 pm and 19:00 pm) and are concentrated between 10:00 am and 23:00 pm, which is consistent with the general operation pattern for the fleet vehicles (electric vehicles). On average, there are 151.5 grids covered in each hour, which accounts for about $4.5 \%$ of the entire study area.

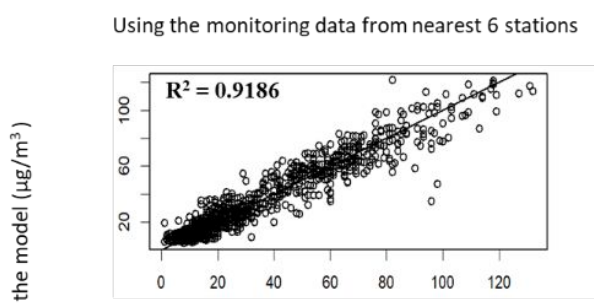

(a)

Using monitoring data from nearest 6 stations and 5 mobile observations, meteorological and road network information

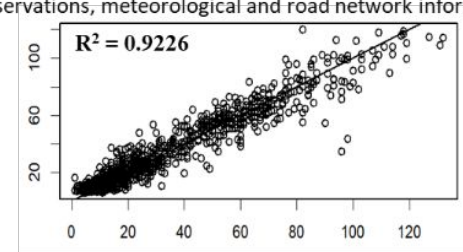

(d)

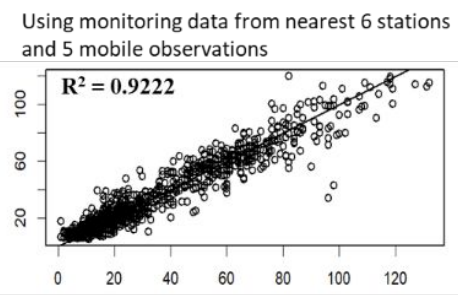

(b)

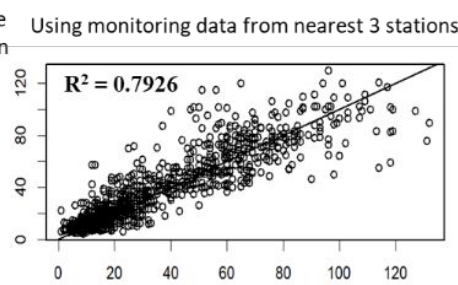

(e)

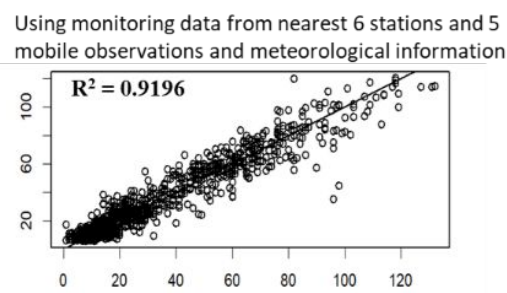

(c)

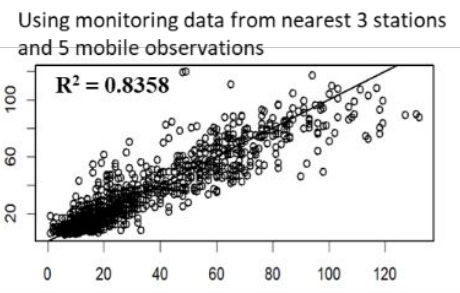

(f)

Benchmark PM2.5 concentration from stationary monitors $\left(\mu \mathrm{g} / \mathrm{m}^{3}\right)$

Fig. S6. Performance of the model only using stationary monitoring data as benchmarks using different predictors: (a) only the monitoring data from the nearest 6 stations; (b) the monitoring data from the nearest 6 stations and 5 mobile observations; (c) the monitoring data from the nearest 6 stations, 5 mobile observations, and meteorological information; (d) the monitoring data from the nearest 6 stations, 5 mobile observations, meteorological and road network information; (e) only the monitoring data from the nearest 3 stations; and (f) the monitoring data from the nearest 3 stations and 5 mobile observations. 


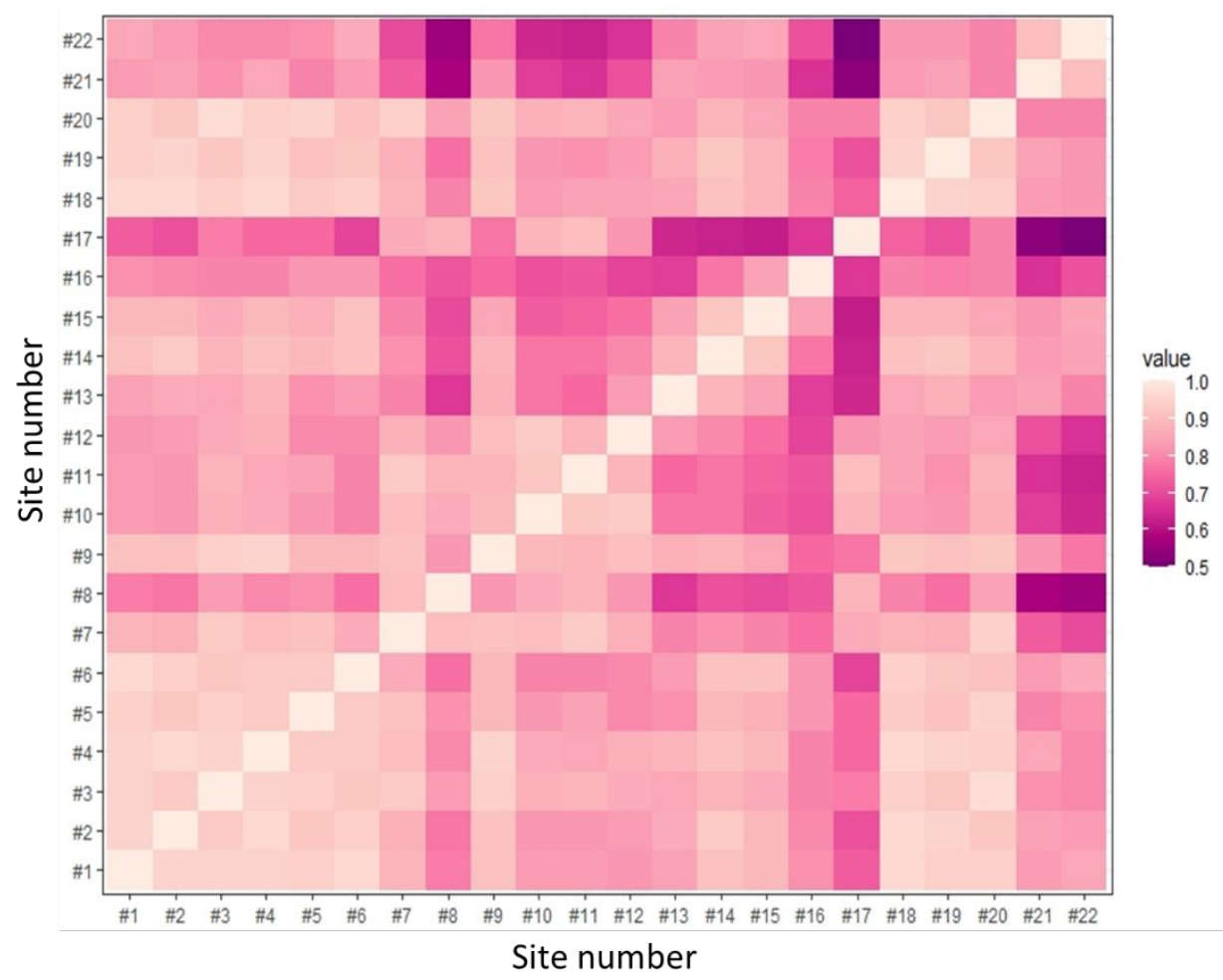

Fig. S7. The correlation between $\mathrm{PM}_{2.5}$ concentrations from fixed-site monitoring stations during the period for the two-phase mobile monitoring campaign (2018.12.22 to 2019.1.26 and 2019.7.2 to 2019.8.11).
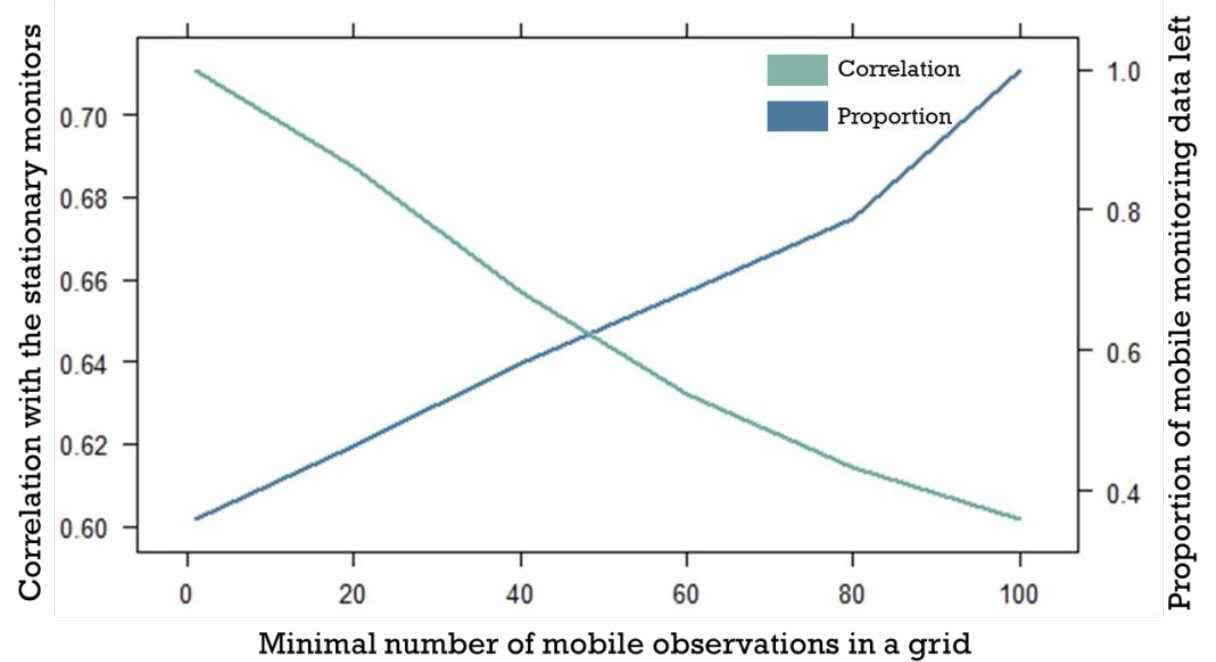

Fig. S8. Data preparation for the mobile monitoring data. We set the minimal number of mobile observations needed in a grid based on their correlation to the monitoring data from stationary monitors in the same grid as well as the number of data left, to ensure the mobile monitoring data to have relative high correlation with the stationary monitoring data and also enough observations. In this study, the aggregated data calculated by less than 40 (3-seconds frequency data) observations (i.e., equivalent of two-minute observation) are removed. 


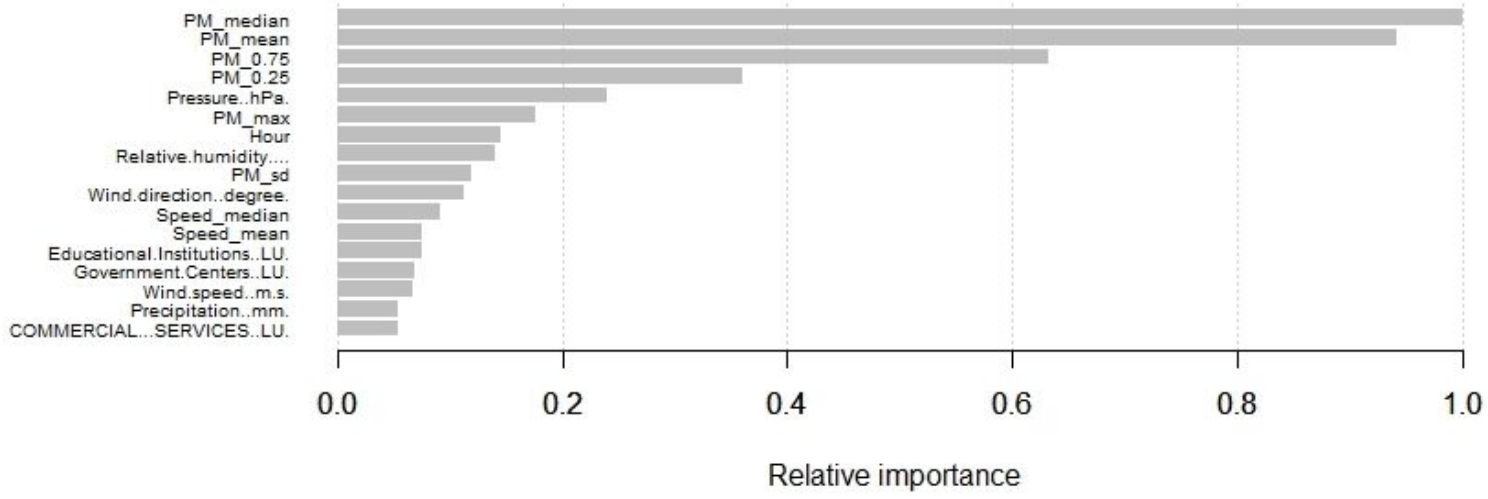

Fig. S9. Relative importance of the variables used in the data transformation model. The variables about the $\mathrm{PM}_{2.5}$ concentration distribution (especially the median, mean, and $3^{\text {rd }}$ quantile) are the most important variables, followed by the meteorological (e.g., pressure, relative humidity, and wind direction) and land use data.

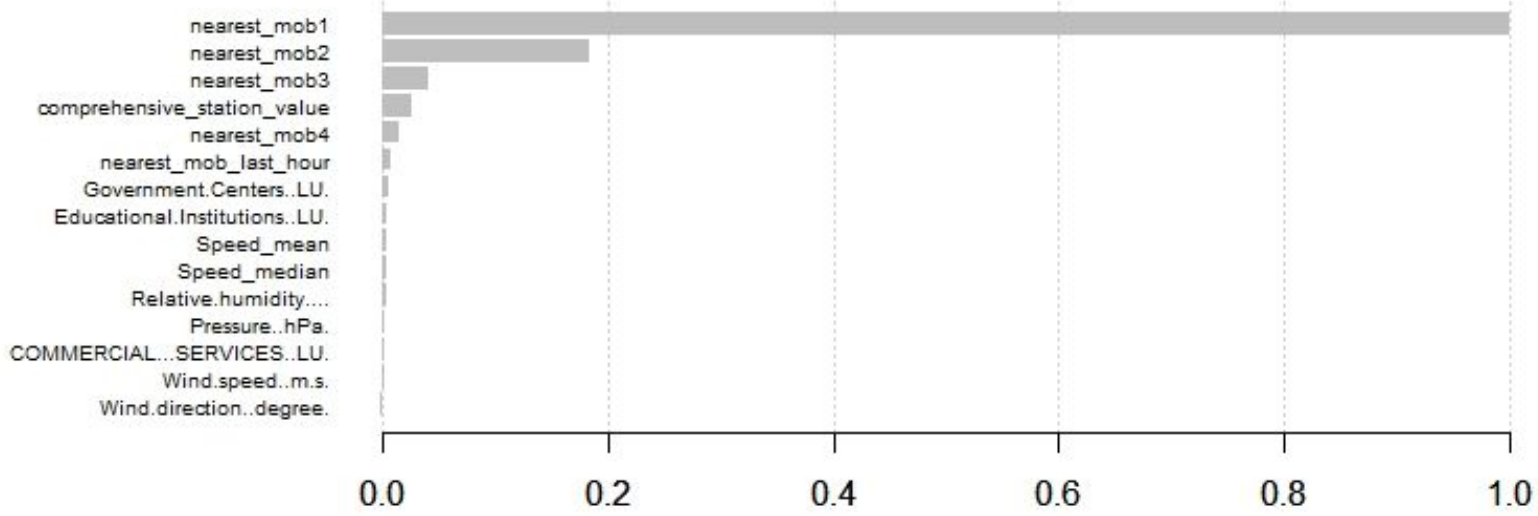

Relative importance

Fig. S10. Relative importance of the variables used in the $\mathrm{PM}_{2.5}$ concentration inference model based on XGBoost. The $\mathrm{PM}_{2.5}$ concentration variables from the nearest two mobile monitoring are the most important variables, followed by the meteorological (e.g., pressure, relative humidity, and wind direction) and land use data. 


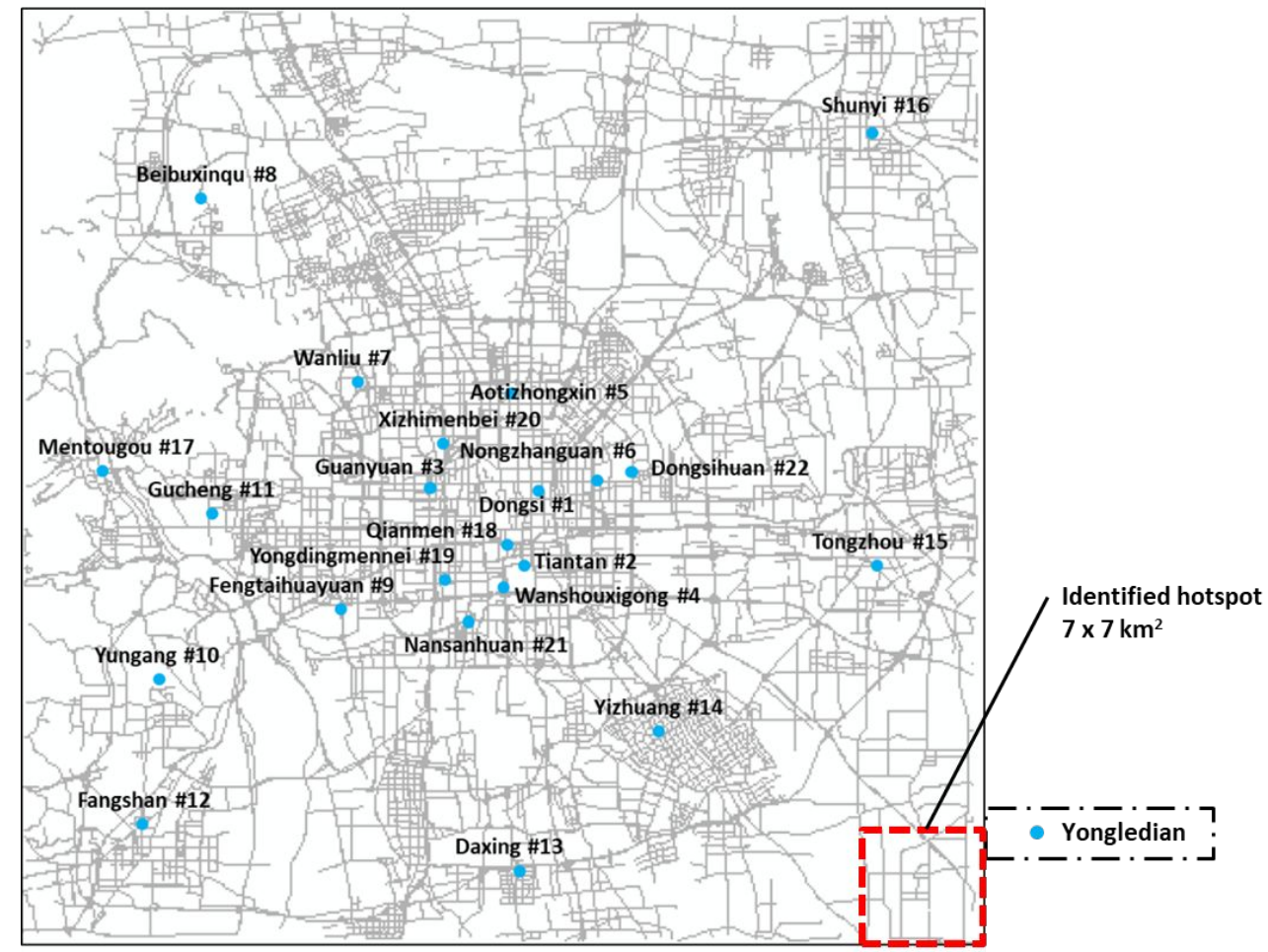

Fig. S11. The location of the nearby monitoring station (Yongledian, LNG: 116.783, LAT: 39.712) and the identified hotspots.

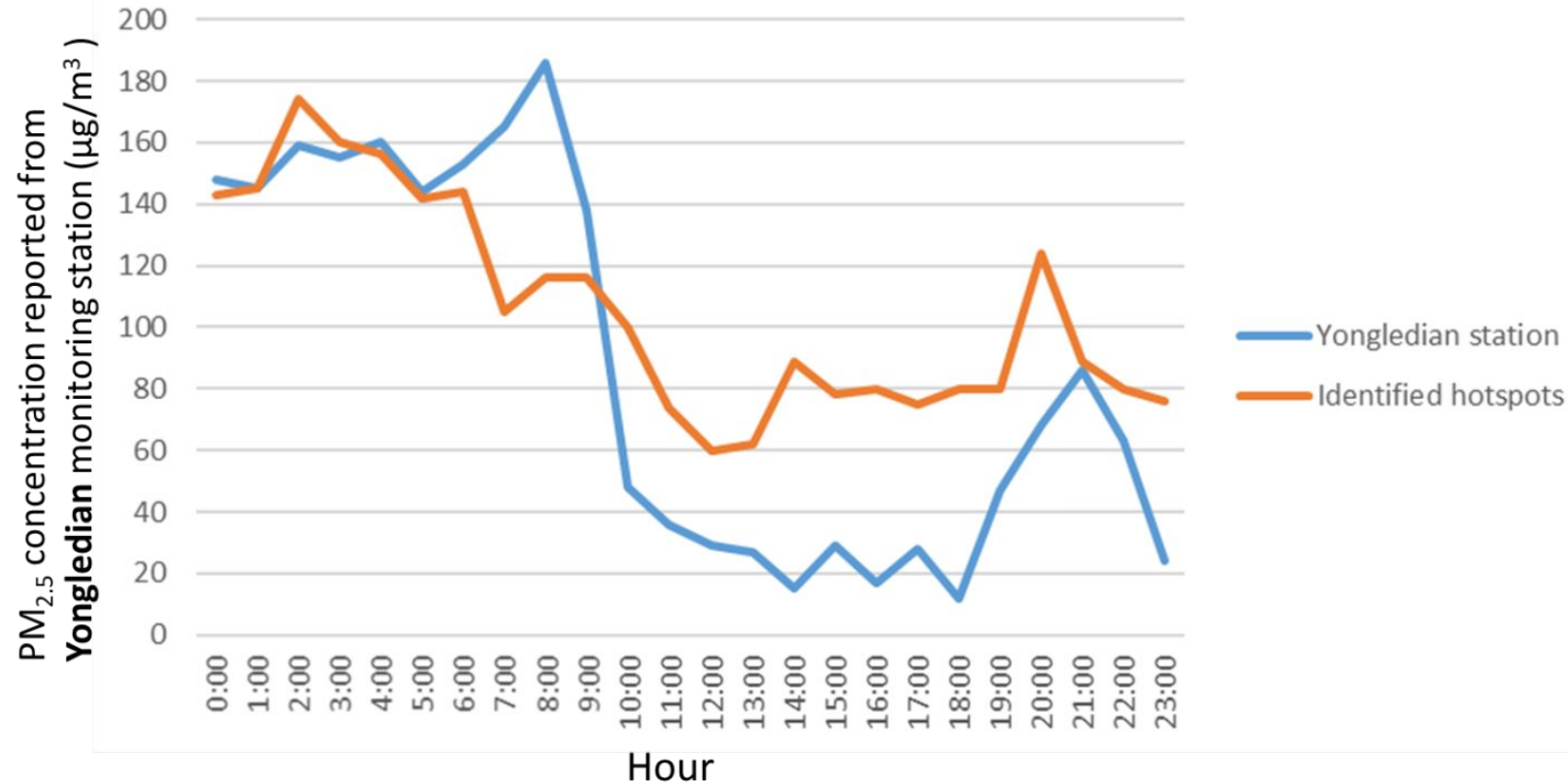

Fig. S12. Hourly $\mathrm{PM}_{2.5}$ concentration reported from Yongledian monitoring station and the average hourly $\mathrm{PM}_{2.5}$ concentration from the identified hotspots on December 31, 2018. 

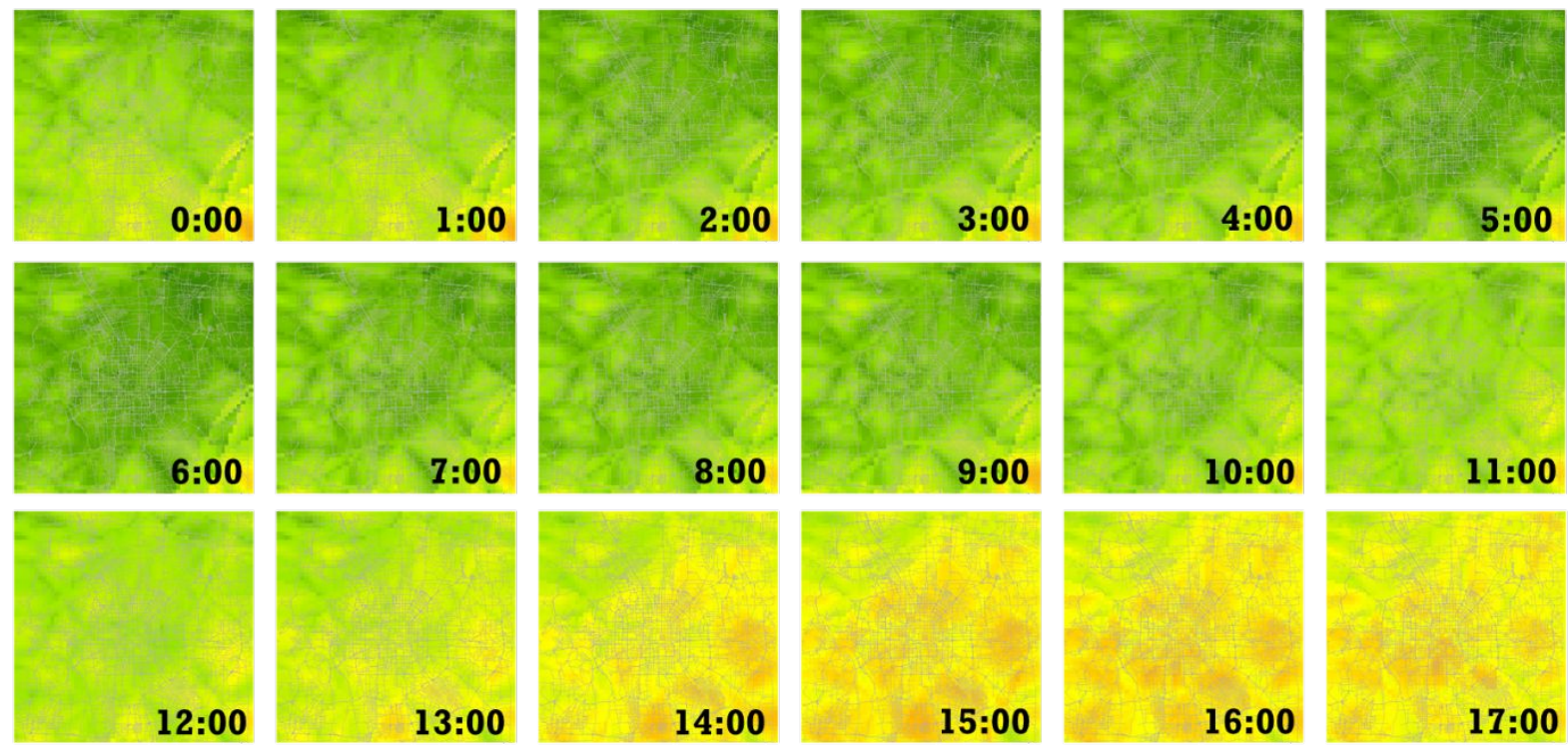

18:00

19:00
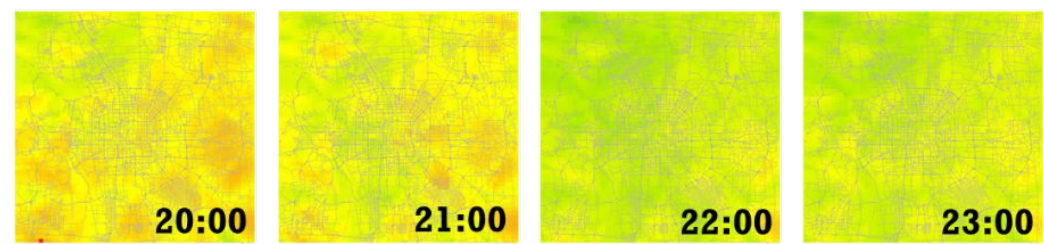

Fig. S13. Hourly $\mathrm{PM}_{2.5}$ concentration distribution on a typical weekday in summer (July 19, 2019) in Beijing inferred by our model demonstrating a transition of air quality from good to moderate pollution. 